\title{
RGS Proteins as Critical Regulators of Motor Function and Their Implications in Parkinson's Disease
}

\author{
Katelin E. Ahlers-Dannen, ${ }^{1}$ Mackenzie M. Spicer, ${ }^{1}$ and Rory A. Fisher \\ Department of Neuroscience and Pharmacology (K.E.A.-D., M.M.S., R.A.F.), lowa Neuroscience Institute (R.A.F.), and \\ Interdisciplinary Graduate Program in Molecular Medicine (M.M.S., R.A.F.), University of lowa Carver College of Medicine, lowa \\ City, lowa
}

Received November 7, 2019; accepted January 25, 2020

\section{ABSTRACT}

Parkinson disease (PD) is a devastating, largely nonfamilial, agerelated disorder caused by the progressive loss of dopamine (DA) neurons in the substantia nigra pars compacta (SNc). Release of DA from these neurons into the dorsal striatum is crucial for regulating movement and their loss causes PD. Unfortunately, the mechanisms underlying SNc neurodegeneration remain unclear, and currently there is no cure for PD, only symptomatic treatments. Recently, several regulator of $G$ protein signaling (RGS) proteins have emerged as critical modulators of PD pathogenesis and/or motor dysfunction and dyskinesia: RGSs 4, 6, 9, and 10. Striatal RGS4 has been shown to exacerbate motor symptoms of DA loss by suppressing $\mathrm{M}_{4^{-}}$ autoreceptor- $\mathrm{G} \alpha_{\mathrm{i} / \mathrm{o}}$ signaling in striatal cholinergic interneurons. RGS6 and RGS9 are key regulators of $D_{2} R-G \alpha_{i / o}$ signaling in SNc DA neurons and striatal medium spiny neurons, respectively. RGS6, expressed in human and mouse SNc DA neurons, suppresses characteristic PD hallmarks in aged mice, including SNc DA neuron loss, motor deficits, and $\alpha$-synuclein accumulation. After DA depletion, RGS9 (through its inhibition of medium spiny neuron $D_{2} R$ signaling) suppresses motor dysfunction induced by L-DOPA or $\mathrm{D}_{2} \mathrm{R}$-selective agonists. RGS10 is highly expressed in microglia, the brain's resident immune cells. Within the SNc, RGS10 may promote DA neuron survival through the upregulation of prosurvival genes and inhibition of microglial inflammatory factor expression. Thus, RGSs $4,6,9$, and 10 are critical modulators of cell signaling pathways that promote SNc DA neuron survival and/or proper motor control. Accordingly, these RGS proteins represent novel therapeutic targets for the treatment of PD pathology.

\section{SIGNIFICANCE STATEMENT}

Parkinson disease (PD), the most common movement disorder, is a progressive neurodegenerative disease characterized by substantia nigra pars compacta (SNc) dopamine (DA) neuron loss and subsequent motor deficits. Current PD therapies only target disease motor symptomology and are fraught with side effects. Therefore, researchers have begun to explore alternative therapeutic options. Regulator of G protein signaling (RGS) proteins, whether primarily expressed in SNc DA neurons (RGS6), striatal neurons (RGSs 4 and 9), or microglia (RGS10), modulate key signaling pathways important for SNc DA neuron survival and/or proper motor control. As such, RGS proteins represent novel therapeutic targets in PD.

\section{Introduction}

Parkinson disease (PD) is a largely nonfamilial, progressive, neurodegenerative disorder characterized by the loss of dopamine (DA) neurons in the substantia nigra pars compacta

This work was supported by the National Institutes of Health [AA025919]; the Michael J. Fox Foundation [1151]; and University of Iowa Pharmacological Sciences Training Grant [T32GM067795].

${ }^{1}$ K.E.A.-D. and M.M.S. indicates co-first authors.

https://doi.org/10.1124/mol.119.118836.
(SNc) (Fahn, 2008; Meissner et al., 2011; Shulman et al., 2011; Mhyre et al., 2012). Under normal conditions, these DA neurons project to the striatum where they release DA onto postsynaptic targets, allowing for proper control of motor behavior. Therefore, loss of SNc DA neurons results in the dysregulation of DA signaling within the nigrostriatal pathway (Fig. 1) and the hallmark motor deficits associated with $\mathrm{PD}$, including bradykinesia, muscle rigidity, and resting tremors. PD is widely considered an idiopathic disorder (Fearnley and Lees, 1991) with identifiable genetic mutations

ABBREVIATIONS: AAV, adeno-associated virus; ACh, acetylcholine; CNS, central nervous system; CREB, cAMP Response Element Binding Protein; DA, dopamine; DEP, disheveled, Egl-10, Pleckstrin homology; DAT, DA transporter; DHEX, DEP helical extension; ${ }_{1} R$, dopamine 1 receptor; $\mathrm{D}_{2} \mathrm{R}$, dopamine 2 receptor; GAP, GTPase-activating protein; GGL, G gamma subunit-like; GPCR, G protein-coupled receptor; L-DOPA, L-3,4-dihydroxyphenylalanine; LPS, lipopolysaccharide; MN9D, mesencephalon DA neuroblastoma cell line; MPTP, methyl-4-phenyl-1,2,3,6tetrahydropyridine; 6-OHDA, 6-hydroxydopamine; PD, Parkinson's disease; PKA, protein kinase A; RGS, regulator of G protein signaling; SNc, substantia nigra pars compacta; $\alpha$-syn, $\alpha$-synuclein; TNF $\alpha$, tumor necrosis factor $\alpha$. 


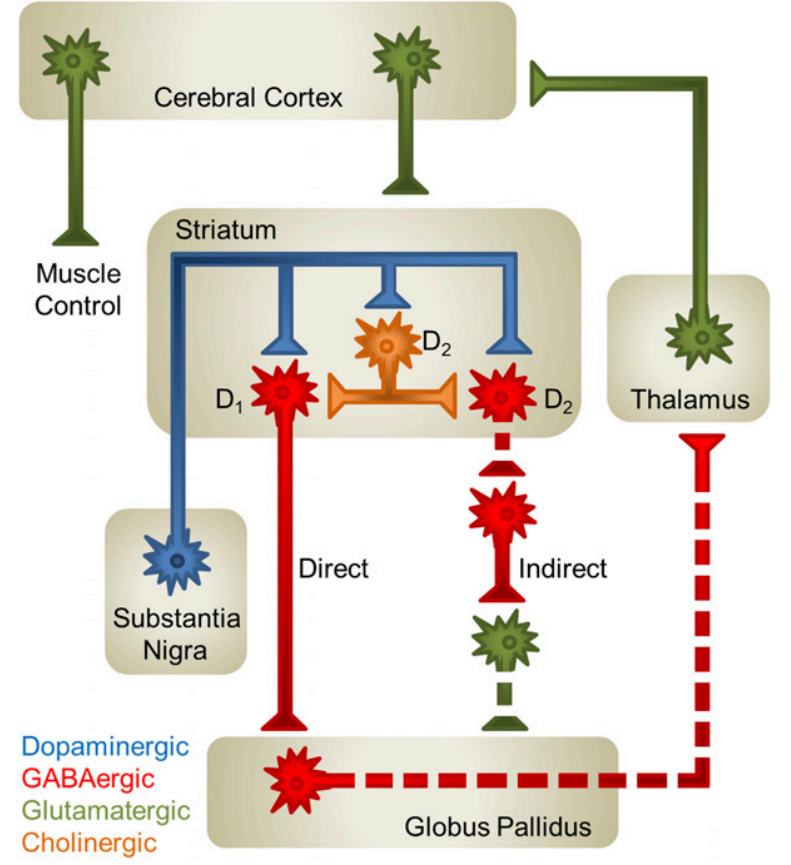

Fig. 1. Model of motor control by the nigrostriatal circuit. DA neurons (blue) originating in the substantia nigra project to the striatum, where they release DA onto $\mathrm{D}_{1} \mathrm{R}$ - and $\mathrm{D}_{2} \mathrm{R}$-containing GABAergic (red) medium spiny neurons and $\mathrm{D}_{2} \mathrm{R}$-containing cholinergic interneurons (orange). DA, through its interaction with the $G \alpha_{\mathrm{s}}$-coupled $\mathrm{D}_{1} \mathrm{R}$, promotes neuronal signaling. In contrast, $\mathrm{D}_{2} \mathrm{R}-\mathrm{G} \alpha_{\mathrm{i} / \mathrm{o}}$ activation ultimately inhibits neuronal signaling. DA release in the striatum silences GABAergic neurons of the globus pallidus (GP) by enhancing the activity of $\mathrm{D}_{1} \mathrm{R}$-containing GABAergic medium spiny neurons (MSNs) of the direct pathway, increasing GABA release into the GP, and by silencing $\mathrm{D}_{2} \mathrm{R}$-containing GABAergic MSNs that begin the indirect pathway, ultimately decreasing glutamate release into the GP. Silencing of GP GABAergic neurons promotes thalamic glutamatergic (green) signaling to the cortex and proper motor control. Solid axons denote pathways that are "on" and dashed axons represent pathways that are "silent."

accounting for only 5\%-9\% of clinical cases (Nussbaum and Ellis, 2003). Currently, the primary risk factor associated with PD is aging (Fearnley and Lees, 1991), with the idiopathic form of the disorder affecting $2 \%$ of the population over 60 and $15 \%$ of those individuals over the age of 85 . Despite decades of research, the molecular characteristics/mechanisms that predispose SNc DA neurons to age-related degeneration remain unknown. As a result, there is currently no cure for PD, only symptomatic treatments.

The prevalence of PD is vast, with nearly 1 million people in the United States suffering from the disease, and it continues to rise as the proportion of elders in our society expands. With 60,000 new PD diagnoses annually, there has been considerable interest in developing novel and improved therapeutic alternatives. Currently, primary therapeutic strategies used to treat PD-related motor symptoms work to correct the dysregulated DA signaling, and include drugs such as levodopa (L-DOPA), a blood-brain barrier-permeable DA precursor, and DA receptor agonists. However, although these drugs are effective in the short-term at correcting PD-related motor deficits, they are fraught with problems of their own, including wearing-off phenomena (Pahwa and Lyons, 2009; Jenner, 2013), dyskinesias (Schrag and Quinn, 2000; Thanvi et al., 2007), and various nonmotor complications (Chaudhuri et al., 2006;
Poewe, 2008). Due to these issues, researchers have begun to explore whether drugs that modulate nondopaminergic neurotransmission systems could be of use in PD. G protein-coupled receptors (GPCRs), the largest family of cell-surface receptors encoded by the genome, have provided promising avenues for alternative PD therapies. In particular, animal models of PD have implicated modulation of noradrenergic, cholinergic, adenosinergic, glutamatergic, and serotonergic neurotransmission as possible adjunctive therapies to current dopaminergic treatments (reviewed by Lemos et al. [2018]). Unfortunately, high levels of conservation in the orthosteric binding site within GPCR subfamilies has limited the development of receptorsubtype specific drugs and thus, like the dopaminergic therapies currently employed in PD, these treatment options would likely have numerous side effects. As such, it is of the utmost importance to identify alternative methods of modulating GPCR signaling within a subfamily. Regulator of $\mathrm{G}$ protein signaling (RGS) proteins may hold the key.

RGS proteins modulate the magnitude and duration of GPCR signaling by facilitating heterotrimeric $\mathrm{G}$ protein inactivation through their GTPase-activating (GAP) activity toward $\mathrm{G} \alpha$ subunits, a function bestowed by their RGS domain. The RGS protein family includes 20 canonical RGS proteins, four of which have been implicated in PD pathogenesis: RGSs 4, 6, 9, and 10 (Fig. 2). Whether they are expressed primarily in SNc DA neurons (RGS6), striatal neurons (RGSs 4 and 9), or the brain's resident immune cells (microglia, RGS10), these RGS proteins have been shown to modulate key signaling pathways that are important for SNc DA neuron survival and/or proper motor control. As such, these RGS proteins represent novel therapeutic targets for the treatment of Parkinson's pathology.

\section{RGS4}

RGS4 is a small $23 \mathrm{kDa}$ member of the R4 family (Fig. 2) selectively expressed in the central nervous system (CNS) and

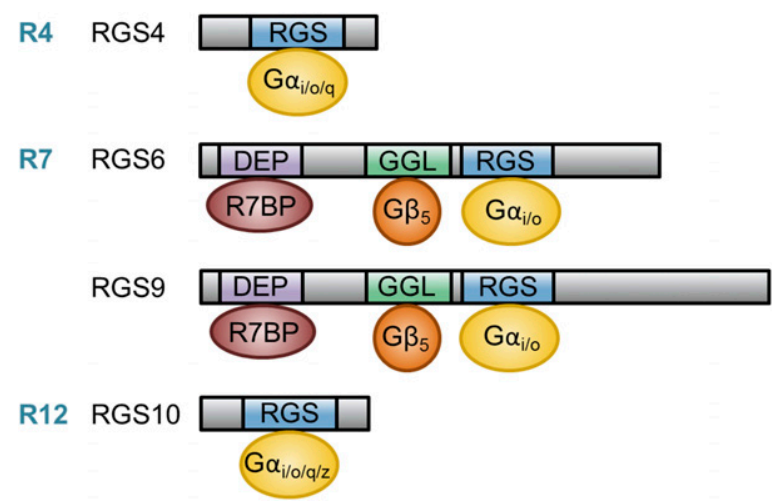

Fig. 2. Comparison of the protein structure and interaction partners for RGS proteins implicated in PD and proper motor function. RGSs 4, 6, 9, and 10 have been implicated in PD pathogenesis and proper motor function. RGS4 is a member of the R4 RGS subfamily that, through its RGS domain, functions as a GAP for $\mathrm{G} \alpha_{\mathrm{j} / \mathrm{o} / \mathrm{q}}$. RGSs 6 and 9 are members of the R7 RGS subfamily that, through their RGS domains, function as GAPs for $\mathrm{G} \alpha_{\mathrm{i} / \mathrm{o}}$. Members of the R7 subfamily are characterized by two unique domains outside of their RGS domain, the DEP/DHEX domain and the GGL domain. The DEP/DHEX domain allows R7 family members bind to the membrane anchor proteins R7BP or R9AP, whereas the GGL domain promotes interaction with $\mathrm{G} \beta_{5}$, which is required for stabilization of all R7 family members. RGS10 is the smallest RGS protein $(\sim 20 \mathrm{kDa})$ and is a member of the R12 RGS subfamily, thus functioning as a GAP for $\mathrm{G} \alpha_{\mathrm{i} / \mathrm{o} / \mathrm{q} / \mathrm{z}}$ 
heart of both humans and rodents (Zhang et al., 1998; Erdely et al., 2004; Bansal et al., 2007). As a member of the R4 family, RGS4 functions as a GAP for $\mathrm{G} \alpha_{\mathrm{q}}$ and $\mathrm{G} \alpha_{\mathrm{i} / \mathrm{o}}$. Within the CNS, RGS4 mRNA is predominantly expressed in the amygdala and striatum but is also present in most cortical neuronal layers (Ebert et al., 2006).

Various studies have implicated striatal RGS4 in the regulation of CNS cholinergic and dopaminergic signaling, as well as endocannabinoid-mediated long-term depression (Geurts et al., 2003; Ding et al., 2006; Lerner and Kreitzer, 2012). In PD, degeneration of SNc DA neurons is accompanied by an increase in striatal acetylcholine (ACh) release, resulting in exacerbated motor symptoms. It was first hypothesized that this increase in cholinergic neurotransmission resulted from reduced $\mathrm{D}_{2} \mathrm{R}$-mediated inhibition of synaptic $\mathrm{Ca}_{\mathrm{v}} 2$ channels in striatal cholinergic interneurons (Fig. 1). However, Ding et al. (2006) demonstrated that although $\mathrm{Ca}_{\mathrm{v}} 2$ activity remained unchanged after DA depletion, its activity was attenuated by the $\mathrm{M}_{4}$ muscarinic autoreceptor expressed on these interneurons.

Given that $\mathrm{M}_{4}$ autoreceptors are $\mathrm{G} \alpha_{\mathrm{i} / \mathrm{o}_{0}}$-coupled, their signaling may be regulated by RGS4 (Fig. 3). Several studies, some conflicting, have examined the expression and function of striatal RGS4 in the context of DA depleted rodent PD models. Geurts et al. (2003) initially described a significant reduction in striatal mRNA expression of both RGS4 and RGS9 after unilateral 6-hydroxydopamine (6-OHDA) lesion or reserpine (adrenergic blocker)-mediated DA depletion. However, Ding et al. (2006) demonstrated the opposite effect of these

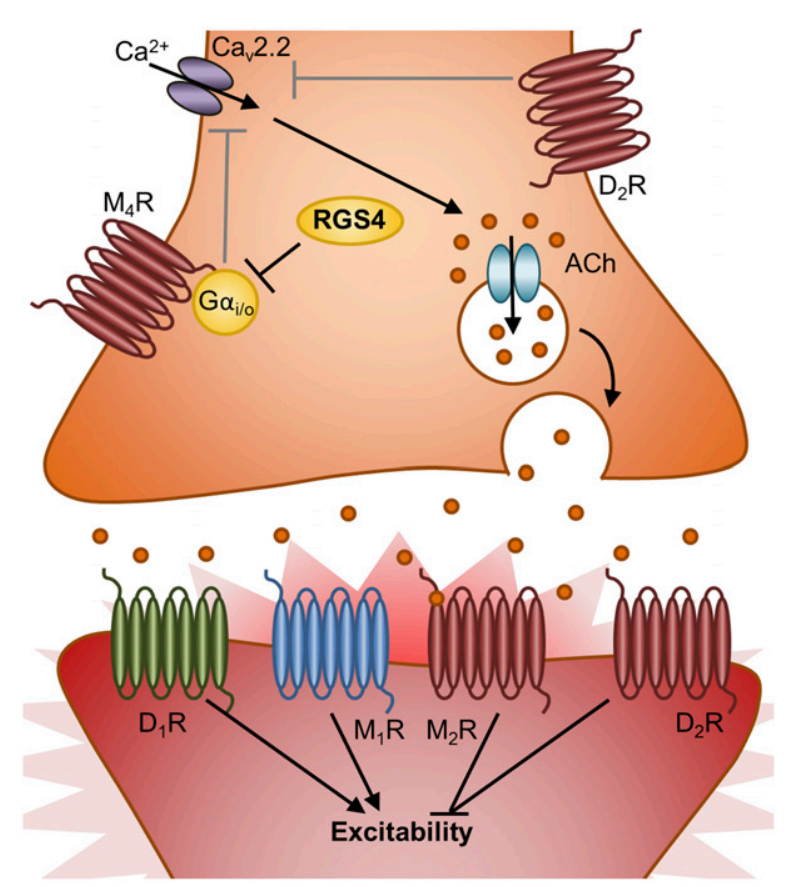

Fig. 3. Model of RGS4's role in regulating striatal ACh release from cholinergic interneurons. RGS4 in striatal cholinergic interneurons inhibits $\mathrm{M}_{4}$ autoreceptor signaling to promote calcium influx through $\mathrm{Ca}_{\mathrm{v}} 2.2$. By preventing striatal cholinergic $\mathrm{M}_{4}$-autoreceptor- $\mathrm{G} \alpha_{\mathrm{i} / \mathrm{o}}$-mediated inhibition of $\mathrm{Ca}_{\mathrm{v}} 2.2$, RGS4 promotes ACh packaging/release. This figure depicts striatal cholinergic interneurons (orange) synapsing on GABAergic $D_{1} R$ and $\mathrm{D}_{2}$ R-containing MSNs (red). These MSNs also likely express $\mathrm{M}_{1}$ and $\mathrm{M}_{2}$ ACh receptors. $\mathrm{G} \alpha_{\mathrm{i} / \mathrm{o}}$-coupled receptors are red, $\mathrm{G} \alpha_{\mathrm{s}}$-coupled receptors are green, and $\mathrm{G} \alpha_{\mathrm{q}}$-coupled receptors are blue. MSN, medium spiny neuron. treatments on striatal RGS4 mRNA expression. In their study, increased RGS4 mRNA expression after either of these treatments was accompanied by a marked attenuation in M4 muscarinic autoreceptor signaling and increased striatal $\mathrm{ACh}$ release. Using intracellular dialysis, they discovered that RGS4 inhibits $\mathrm{M}_{4}$ autoreceptor-mediated $\mathrm{Ca}_{\mathrm{v}} 2$ activity in striatal cholinergic interneurons (Fig. 3). In agreement with these findings, Ko et al. (2014) reported not only elevated RGS4 expression in L-DOPA treated 6-OHDA-lesioned rats but also that RGS4 mRNA suppression during L-DOPA priming attenuated the development of drug-induced dyskinesia. Similarly, RGS4 ${ }^{-1-}$ mice exhibited fewer motor behavioral deficits after 6-OHDA lesion (Lerner and Kreitzer, 2012). However, another study reported that RGS4 ${ }^{-1-}$ mice are not protected from 6-OHDA-induced injury and motor dysfunction (Ashrafi et al., 2017).

Collectively, these studies (Ding et al., 2006; Ko et al., 2014) suggest RGS4 inhibition may be useful in treating druginduced dyskinesias in PD. In light of this, Blazer et al. (2015) described the selectivity of a thiadiazolidinone inhibitor (CCG-203769) they discovered for RGS4 over other RGS proteins that might represent a novel therapeutic option for PD. However, RGS4 has not been implicated in modulating SNc DA neuron loss, and it remains unclear whether it promotes motor deficits after DA depletion through its striatal actions.

\section{RGS6}

RGS6 is a member of the R7 RGS subfamily, which modulates $\mathrm{G} \alpha_{\mathrm{i} / \mathrm{o}}$ signaling (Hooks et al., 2003) and shares two unique domains in addition to the RGS domain: the disheveled, Egl-10, Pleckstrin homology (DEP)/DEP helical extension (DHEX) domain and the G gamma subunit-like (GGL) domain. The DEP/DHEX domain allows R7 family members to associate with the membrane anchor proteins R7BP and R9AP (Martemyanov et al., 2005; Drenan et al., 2006), whereas the GGL domain promotes interaction with the atypical $\mathrm{G} \beta$ subunit, $\mathrm{G} \beta_{5}$, which is required for stabilization of all R7 family members (Fig. 2) (Posner et al., 1999; Snow et al., 1999; Witherow et al., 2000; Chen et al., 2003; Narayanan et al., 2007; Cheever et al., 2008; Porter et al., 2010).

RGS6 is expressed in a wide variety of tissues throughout the body (Gold et al., 1997; Yang et al., 2010; Maity et al., 2011, 2012; Bifsha et al., 2014; Stewart et al., 2014, 2015), with highest mRNA and protein levels expressed in the brain. When Chatterjee et al. (2003) first cloned RGS6 using a Marathon-ready human brain cDNA library, they described multiple RGS6 splice variants predicted to produce 36 distinct RGS6 protein isoforms containing either long (RGS6L, $\sim 49-56 \mathrm{kDa}$ ) or short (RGS6S, $\sim 32-40 \mathrm{kDa})$ N-terminal domains, an incomplete or intact GGL domain, and nine alternative C-terminal sequences. Although sequence similarities have complicated the study of individual RGS6 protein isoforms, Bifsha et al. (2014) and Luo et al. (2019) have demonstrated that RGS6L isoforms may be key survival factors for SNc DA neurons.

RGS6 was first implicated in PD when it was discovered that RGS6 was the most differentially lost gene in ventral SNc DA neurons in a developmental PD model, Pituitary homeobox 3-deficient mice (Bifsha et al., 2014). Subsequent 
immunohistochemical analyses revealed that RGS6 was exclusively expressed in DA neurons within the SNc of both mice and humans that are lost with PD (Bifsha et al., 2014; Luo et al., 2019). Comparative phenotyping of $\mathrm{RGS}^{+/+}$and RGS6 ${ }^{-/-}$mice revealed that RGS6 acts as a critical survival factor for SNc DA neurons that, when lost, results in their late-age degeneration (Bifsha et al., 2014), as well as PD-like motor deficits, including reduced mobility (open field test and Rotarod) and abnormal gait (DigiGait analysis) (Luo et al., 2019). In aged RGS6 ${ }^{-/-}$mice, SNc DA neuron degeneration is associated with markers of pathologic change (Fluoro-Jade $\mathrm{C}$ and Nissl staining) as well as reduced levels of the DA precursor synthesizing enzyme, tyrosine hydroxylase, and the vesicular DA transporter, Vmat2. In addition, SNc DA neuron degeneration is accompanied by enhanced $\mathrm{D}_{2}$-autoreceptor signaling, increased expression of the DA transporter (DAT) (Bifsha et al., 2014), and increased sensitivity of RGS6 ${ }^{-/-}$mice to quinpirole $\left(\mathrm{D}_{2} \mathrm{R}\right.$ agonist) suppression of locomotion (Luo et al., 2019), known to be mediated by the nigrostriatal $\mathrm{D}_{2}$-autoreceptor (Usiello et al., 2000; Wang et al., 2000; Lindgren et al., 2003; Bello et al., 2011). All of these molecular changes likely contribute to the dysregulated production and release/reuptake of $\mathrm{DA}$ in the nigrostriatal circuit of aged $\mathrm{RGS}^{-1-}$ mice, cytotoxic DA byproduct (3,4-dihydroxyphenylacetaldehyde) accumulation, and the observed PD-like motor deficits (Fig. 4) (Luo et al., 2019).

The expression of several genes that had previously been associated with PD, such as: DJ-1 (PARK7), PINK1 (PARK6), LRRK2 (PARK8), and SNCA ( $\alpha$-synuclein [ $\alpha$-syn]) were also altered in $\mathrm{RGS6}^{-/-}$mice (Bifsha et al., 2014). Of particular interest, immunohistochemical analysis of aged $\mathrm{RGS6}^{-1-}$ mice revealed that they exhibited abnormally high levels of the $\alpha$-syn protein (Luo et al., 2019), a hallmark of PD that is believed to contribute to neurodegeneration (Spillantini et al., 1997; Spillantini et al., 1998; Masliah et al., 2000; Giasson et al., 2002; Singleton et al., 2003; Chartier-Harlin et al., 2004; Li et al., 2004; Chu and Kordower, 2007; Stefanis, 2012; Kim, 2013). The $\alpha$-syn protein observed in aged $\mathrm{RGS}^{-1-}$ mice, unlike that observed in young or wild-type animals, was primarily extracellular and, as revealed by western analysis, highly oligomeric (Luo et al., 2019).

RGS6 likely suppresses late-age-onset SNc DA neuron death and $\alpha$-syn accumulation through its negative regulation of the $\mathrm{SNc} \mathrm{D}_{2}$-autoreceptor-G $\alpha_{\mathrm{i} / \mathrm{o}}$-cAMP/PKA signaling axis (Fig. 4) (Luo et al., 2019). Neuronal cAMP/PKA levels are controlled by GPCRs coupled to either $\mathrm{G} \alpha_{\mathrm{s}}$ or $\mathrm{G} \alpha_{\mathrm{i} / \mathrm{o}}$, which function to increase or decrease cAMP, respectively. Mittal et al. (2017) discovered that $\beta$-agonists, which signal through $\mathrm{G} \alpha_{\mathrm{s}}$-linked $\beta$-adrenergic receptors, dramatically reduce both $\alpha$-syn expression and human PD incidence while also inhibiting 1-methyl-4-phenyl-1,2,3,6-tetrahydropyridine (MPTP)-induced SNc DA neuron loss in mice. RGS6 $^{-/-}$mice exhibit hyperactive SNc $\mathrm{D}_{2}$ autoreceptor$\mathrm{G} \alpha_{\mathrm{i} / \mathrm{o}}$ signaling and reduced SNc DA neuron PKA signaling (Luo et al., 2019), suggesting that RGS6 likely regulates SNc DA neuron survival and $\alpha$-syn expression through cAMPmediated mechanisms as well.

In summary, the findings of Bifsha et al. (2014) and Luo et al. (2019) are significant because they reveal that the loss of a single gene, RGS6, phenocopies late-age-onset $\mathrm{PD}$ in mice (Fig. 4).

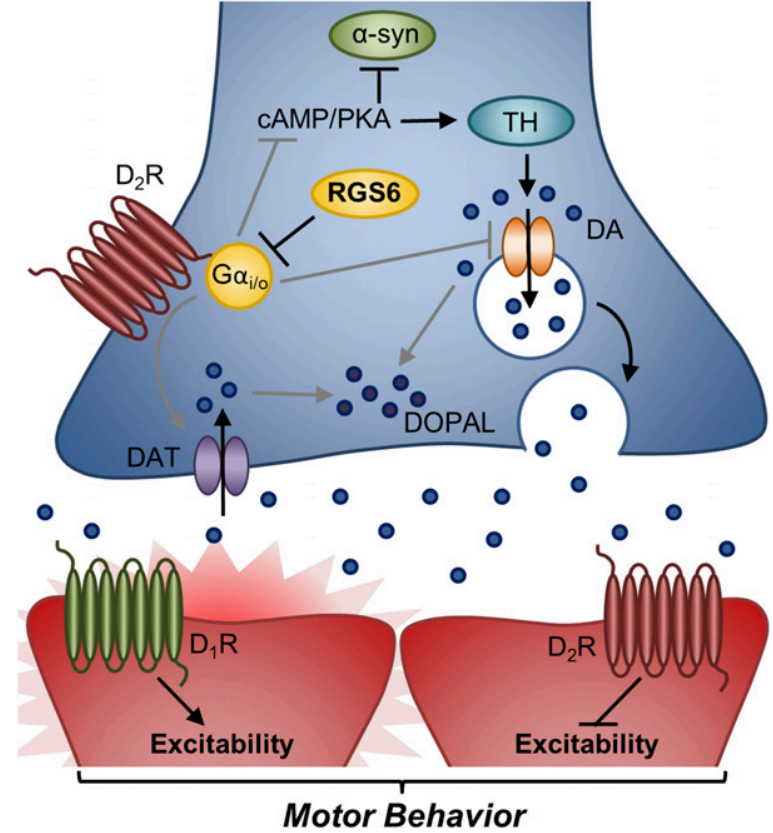

Fig. 4. Model of RGS6's role in regulation of $\mathrm{SNc} \mathrm{D}_{2}$ autoreceptor signaling. RGS6 in SNc DA neurons inhibits $\mathrm{D}_{2}$-autoreceptor signaling to promote proper DA homeostasis and neurotransmission as well as prevent aberrant $\alpha$-synuclein accumulation. By inhibiting $\mathrm{SNc}_{2} \mathrm{D}_{2}$-autoreceptor-G $\alpha_{\mathrm{i} / \mathrm{o}}$ signaling, RGS6 promotes DA packaging/release by preventing Vmat2 downregulation and DAT upregulation/activation. In addition, RGS6 inhibition of $\mathrm{D}_{2}$-autoreceptor- $\mathrm{G} \alpha_{\mathrm{i} / \mathrm{o}}$ signaling promotes cAMP/PKA signaling, increasing DA synthesis ( $\mathrm{TH}$ phosphorylation) and suppressing $\alpha$-synuclein expression. $\beta$-agonists, which have been shown to reduce PD incidence work in a similar fashion. In contrast, RGS6 loss, as seen in the RGS6 $^{-1-}$ mouse model, disinhibits SNc $D_{2}$-autoreceptor-G $\alpha_{\mathrm{i} / \mathrm{o}}$ signaling, reducing cAMP-mediated DA synthesis and increasing $\alpha$-synuclein accumulation. Furthermore, RGS6 loss is associated with cytotoxic DA (DOPAL) accumulation due to $\mathrm{SNc} \mathrm{D}_{2}$-autoreceptor-G $\alpha_{\mathrm{i} / \mathrm{o}}$-mediated Vmat2 downregulation and DAT upregulation. This figure depicts SNc DA neurons (blue) synapsing on GABAergic $D_{1} R$ - and $D_{2} R$-containing MSNs (red). $\mathrm{G} \alpha_{\mathrm{i} / \mathrm{o}}$-coupled receptors are red and $\mathrm{G} \alpha_{\mathrm{s}}$-coupled receptors are green. DOPAL, 3,4-dihydroxyphenylacetaldehyde; MSN, medium spiny neuron; $\mathrm{TH}$, tyrosine hydroxylase.

\section{RGS9}

RGS9 is a member of the R7 RGS protein subfamily and therefore, like RGS6, modulates $\mathrm{G} \alpha_{\mathrm{i} / \mathrm{o}}$ signaling (Hooks et al., 2003), contains the DEP/DHEX and GGL domains and is stabilized by $\mathrm{G} \beta_{5}$ (Fig. 2) (Witherow et al., 2000; Chen et al., 2003; Cheever et al., 2008; Porter et al., 2010). RGS9 exists as two isoforms: RGS9-1, a $\sim 56 \mathrm{kDa}$ protein expressed in the retina (He et al., 1998), and RGS9-2, a $\sim 77 \mathrm{kDa}$ protein largely expressed in the striatum (Gold et al., 1997; Thomas et al., 1998; Rahman et al., 1999; Zhang et al., 1999; Liou et al., 2009).

RGS9 was first implicated in PD when Tekumalla et al. (2001) reported elevated RGS9 protein expression in the striatum of patients with PD. One complication of this study was that patients had received L-DOPA, which made it difficult to determine whether the elevation in RGS9 expression was the result of the disease or its treatment. This issue remained unclarified until Geurts et al. (2003) reported that rat striatal RGS9 mRNA expression was reduced after 6OHDA nigrostriatal lesioning, a finding that was further corroborated by Kovoor et al. (2005) who reported that $\mathrm{RGS9}^{-1-}$ mice were more susceptible to 6-OHDA-induced $\mathrm{PD}$ than $\mathrm{RGS9}^{+/+}$mice. 
In the striatum, RGS9-2 inhibits $\mathrm{D}_{2} \mathrm{R}-\mathrm{G} \alpha_{\mathrm{i} / \mathrm{o}}$ signaling to modulate motor function (Fig. 5), as demonstrated through investigation of the impact of $D_{1} R$ - and $D_{2} R$-selective agonists on circling behavior of rats overexpressing RGS9-2 in the ventral striatum (nucleus accumbens) (Rahman et al., 2003). Unilateral striatal overexpression of RGS9-2 and treatment with apomorphine or quinpirole $\left(D_{2} R\right.$-selective agonists) induced a strong directional rotation bias toward the side of RGS9-2 overexpression. In contrast, treatment with the $\mathrm{D}_{1} \mathrm{R}$-selective agonist (SKR81297) did not induce a directional rotation bias. Because the authors had demonstrated that RGS9-2 can regulate DA signaling in the basal ganglia, they subsequently wanted to determine the impact of RGS9-2 loss on locomotion and DA receptor expression. Therefore, they performed locomotor analyses on RGS9 ${ }^{-1-}$ mice. These analyses revealed that RGS9 loss is associated with an enhanced locomotor response to amphetamine despite $\mathrm{D}_{1} \mathrm{R}$ and $\mathrm{D}_{2} \mathrm{R}$ expression levels remaining unaltered in the ventral striatum (Rahman et al., 2003).

Kovoor et al. (2005) bolstered these findings by revealing that, although $\mathrm{RGS9}^{-/-}$mice display normal locomotory behavior, they exhibit severe abnormal involuntary movements after treatment with reserpine (adrenergic blocker) in combination with quinpirole or apomorphine. In contrast, reserpine in combination with the $\mathrm{D}_{1} \mathrm{R}$-selective agonist did not induce abnormal movement behaviors. Similarly, RGS9-2 overexpression in striatum of monkeys with a MPTP lesion reduced the incidence of L-DOPA-induced dyskinesia, without minimizing L-DOPA's antiparkinsonian effects (Gold et al., 2007).

In summary, these findings indicate that RGS9 plays a critical role in modulating motor movement through its ability to inhibit $\mathrm{D}_{2} \mathrm{R}-\mathrm{G} \alpha_{\mathrm{i} / \mathrm{o}}$ signaling in the striatum. These findings implicate a postsynaptic role of RGS9 (Fig. 5) versus

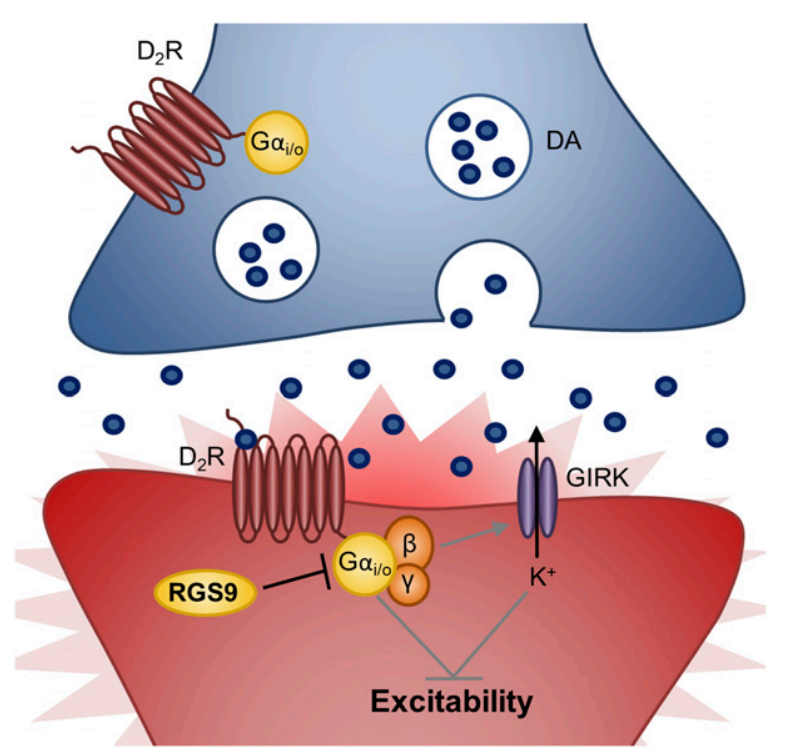

Fig. 5. Model of RGS9's role in regulation of striatal $D_{2} R s$ to promote proper motor control. RGS9 inhibits $\mathrm{D}_{2} \mathrm{R}$ signaling in striatal indirect MSNs (iMSNs) promoting neuronal activity/excitability to regulate motor function. By inhibiting striatal iMSN $\mathrm{D}_{2} \mathrm{R}-\mathrm{G} \alpha_{\mathrm{i} / \mathrm{o}}$ signaling, RGS9 suppresses $\beta \gamma$-mediated GIRK channel activation, promoting depolarization and neuronal firing. This figure depicts SNc DA neurons (blue) synapsing on GABAergic $\mathrm{D}_{2} \mathrm{R}$-containing iMSNs (red). $\mathrm{G} \alpha_{\mathrm{i} / \mathrm{o}}$-coupled receptors are red. MSN, medium spiny neuron. the presynaptic role of RGS6 (Fig. 4) in controlling nigrostriatal movement.

\section{RGS10}

RGS10 is a small $20 \mathrm{kDa}$ member of the R12 RGS subfamily (Fig. 2) that functions as a GAP for $\mathrm{G} \alpha_{\mathrm{i} / \mathrm{o} / \mathrm{q} / \mathrm{z}}$ (Hunt et al., 1996) and is highly expressed in brain regions associated with higher brain function, including the hippocampus, striatum, and dorsal raphe (Gold et al., 1997). RGS10 is unique among the RGS proteins we have discussed thus far in that it is highly expressed in the brain's resident immune cells, microglia, in addition to its low neuronal expression (Fig. 6) (Waugh et al., 2005).

Microglia and neuroinflammation have been widely implicated in PD pathogenesis (reviewed by Joers et al. (2017), Subhramanyam et al. (2019)). Not only are reactive/activated microglia and the inflammatory mediators they produce observed in the brains of patients with PD, but various PDassociated chemicals and neurotoxins, such as 6-OHDA, MPTP, and bacterial lipopolysaccharides (LPS), are known to cause microglial activation and may at least partially induce neuronal cell death through this activation. Furthermore, $\alpha$-syn may sensitize DA neurons to inflammationinduced cell death as well as activate microglia and be modified by microglia, subsequently promoting DA neuron death. Finally, it has been shown that nonsteroidal antiinflammatory drugs may lower the incidence of PD and inhibition of tumor necrosis factor $\alpha(\mathrm{TNF} \alpha)$ or the LPS receptor (toll-like receptor 4) may reduce DA neuron death.

In addition to contributing to PD progression, there is evidence suggesting that inflammation may be one initiating factor in PD (reviewed by (Tansey and Goldberg, 2010)). Aging, the greatest and least understood risk factor associated with idiopathic $\mathrm{PD}$, is known to prime microglia toward activation, resulting in exacerbated inflammation. This priming process and the resulting increased inflammation are predicted to be particularly detrimental in areas of the brain that contain a higher density of microglia, such as the midbrain, and to neuronal populations undergoing a high degree of oxidative processes, such as DA neurons. Supporting this prediction that midbrain DA neurons may be particularly susceptible to microglial priming/activation and inflammation, viruses/conditions associated with inflammation, such as influenza (influenza pandemic 1914-1918), Japanese encephalitis virus exposure, and Crohn's disease, have all been associated with increased PD risk.

Because RGS10 is enriched in microglia and a mutation in the RGS10 gene had already been linked to neurodegenerative age-related maculopathy (Jakobsdottir et al., 2005; Schmidt et al., 2006), Lee et al. (2008) hypothesized that RGS10 loss could predispose an organism to PD, possibly through exaggerated microglial activation. In this initial investigation, Lee et al. (2008) demonstrated that RGS10 ${ }^{-/-}$ mice suffer from increased CNS microglial burden (seen on mixed 129/C57/BL6 background but not on pure C57/BL6 background [Kannarkat et al., 2015]) and activation. Furthermore, RGS $10^{-1-}$ mice were particularly susceptible to LPSmediated SNc DA neuron degeneration. Consistent with this finding and the known role of LPS in microglia inflammatory induction, primary microglia isolated from $\mathrm{RGS}^{-10^{-1}}$ mice had dysregulated inflammatory gene expression profiles 


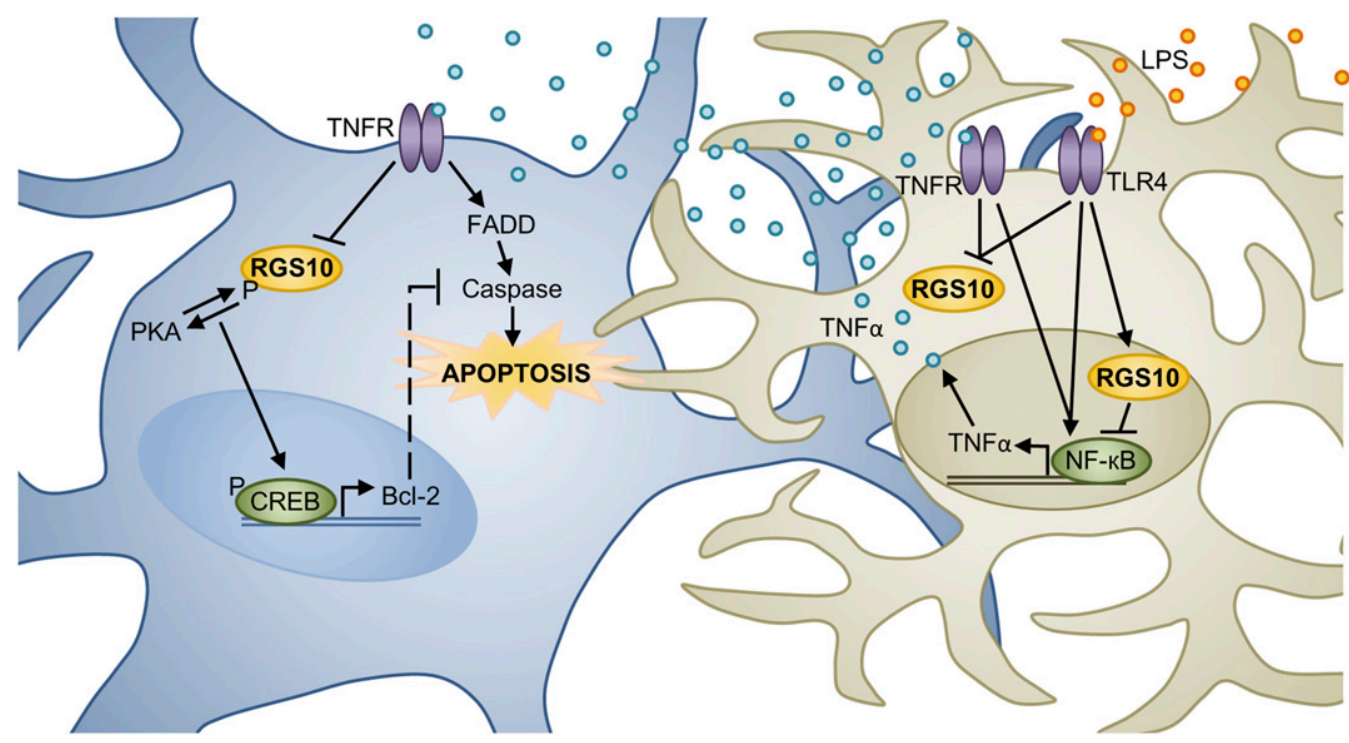

Fig. 6. Model of RGS10's role in modulating SNc DA neuron survival. TNF $\alpha$, an inflammatory factor, can induce neuronal cell death through activation of the TNFR-Fas-associated protein with death domain (FADD)-caspase pathway. Cell culture studies suggest that PKA phosphorylated RGS10 directly promotes MN9D DA cell survival by potentiating PKA-mediated CREB activation and prosurvival gene (Bcl-2) expression. However, RGS10 may also promote cell survival indirectly by inhibiting TNFR/TLR4 (LPS receptor)-NF-kB-mediated inflammatory factor (i.e., TNF $\alpha$ ) expression by microglia, the brain's resident immune cells. Whether acting directly or indirectly to promote DA cell survival, TNF $\alpha$ works to counteract RGS10's positive effects by reducing its expression. This diagram depicts a microglial cell (tan) in close association with a DA neuron (blue) in the SNc. However, the role of RGS10 SNc DA neurons in vivo has yet to be directly examined. FADD, Fas-associated protein with death domain; LPS, bacterial lipopolysaccharides; TLR4, toll-like receptor 4.

under basal conditions and after LPS stimulation. Similar results were obtained from the BV2 murine microglia cell line after RGS10 knockdown. Interestingly, Lee et al. 2008 also demonstrated that culture media taken from LPS-treated BV2 cells induced MN9D (mesencephalon DA neuroblastoma cell line) cell death, which could be prevented by the TNF receptor decoy etanercept. Together, these results suggest that RGS10 functions as a prosurvival factor in PD by inhibiting microglia activation/inflammatory factor production and subsequent DA neuron cell death. However, Lee et al. argued that RGS10 not only promoted DA neuron survival indirectly but also directly as RGS10 ablation in MN9D cells sensitized them to the toxic effects of LPS-treated BV2 cell media, an effect that was once again prevented by etanercept (Fig. 6).

In subsequent publications, Lee et al. $(2011,2012)$ provided further mechanistic insight into the findings described above. In their publication, Lee et al. (2008) demonstrated that RGS10 translocates from the cytoplasm into the nucleus of primary microglia after LPS exposure. Therefore, they hypothesized that, in addition to regulating $\mathrm{GPCR}-\mathrm{G}_{\mathrm{i} / \mathrm{o}}$ signaling, RGS10 may also limit proinflammatory factor expression by inhibiting NF-KB (Lee et al., 2011). In support of this hypothesis, NF- $\mathrm{KB}$ subunit (p65 and p50) expression and transcriptional activity (NF-kB-luciferase reporter plasmid) were significantly increased in RGS10 $0^{-1-}$ primary microglia after TNF $\alpha$ and/or LPS exposure. Furthermore, re-expression of RGS10 in RGS10 ${ }^{-1-}$ primary microglia reduced LPSstimulated inflammatory factor expression (i.e., $\mathrm{TNF} \alpha$ ) and media toxicity toward MN9D cells. Because $\mathrm{TNF} \alpha$ is required for SNc DA neuron degeneration after 6-OHDA administration (McCoy et al., 2006, 2008) and RGS10 appears to modulate microglia TNF $\alpha$ production, Lee et al. (2011) performed viral expression experiments to determine whether RGS10 overexpression could protect against 6-OHDA lesioning.
Compared with 6-OHDA/lenti-GFP-injected rats, rats injected with 6-OHDA/lenti-RGS10 virus displayed significantly decreased microglial activation and DA neuron degeneration (Lee et al., 2011). Together, these results indicate that RGS10 promotes DA neuron survival indirectly by inhibiting microglial NF-kB-mediated expression of proinflammatory factors, particularly $\mathrm{TNF} \alpha$ (Fig. 6). In a later publication, Lee et al. (2012) described the direct prosurvival role of RGS10 in DA neurons. They reported that $\mathrm{TNF} \alpha$ reduces MN9D RGS10 protein expression and that stable overexpression of wild-type RGS10, but not the RGS10-S168A (RGS10SA, resistant to PKA phosphorylation) mutant, in the MN9D cells reduced $\mathrm{TNF} \alpha$-toxicity. This reduction in $\mathrm{TNF} \alpha$-toxicity may relate to RGS10's ability to potentiate PKA-CREB-mediated prosurvival gene (Bcl-2) expression. Together, these results indicate that PKA-mediated RGS10 phosphorylation and RGS10's subsequent promotion of PKA-CREB signaling may underly DA neuron survival (Fig. 6).

In summary, the work by Lee et al. (2008, 2011, 2012) suggests that RGS10 may directly promote DA neuron survival by potentiating PKA-mediated CREB phosphorylation and prosurvival gene expression, as well as indirectly by inhibiting NF-kB-mediated inflammatory factor expression (Fig. 6). Finally, this group has now published evidence suggesting that RGS10 expression may be reduced with age, causing dysregulation of immune/inflammatory pathways that could possibly contribute to PD initiation (Kannarkat et al., 2015).

\section{Conclusion}

The studies described here suggest that RGSs 4, 6, 9, and 10 are critical modulators of both $G$ protein-dependent and -independent cell signaling pathways that promote SNc DA neuron survival and/or proper motor control. Together, these 
findings implicate RGSs 4, 6, 9, and 10 as novel therapeutic targets for the treatment of PD pathology, not just symptomology. Although the role of these RGS proteins in PD has clearly been established through genetic animal models, at present, they cannot be tested pharmacologically. Indeed, currently identified RGS protein inhibitors are not selective (Hayes et al., 2018; O’Brien et al., 2019). In addition, these inhibitors lack required tissue and neuronal specificity and likely will affect multiple tissues where these RGS proteins are expressed. Finally, no drugs have been identified that increase the activity of any member of the RGS protein family as would be needed to target RGSs 6, 9, and 10 for PD treatment.

Currently, the majority of pharmaceuticals targeting GPCR signaling disrupt the pathway at the extracellular ligand-GPCR interface (reviewed by Neubig and Siderovski [2002]). Although these pharmacological therapies successfully inhibit GPCR signaling, they often lack tissue specificity. Similarly, the search for selective RGS inhibitors and/or activators has proven difficult (Neubig and Siderovski, 2002; Hayes et al., 2018; O'Brien et al., 2019). In evidence of this, Hayes et al. (2018) recently demonstrated that each of 13 identified RGS4 inhibitors inhibited other members of the RGS protein family, sometimes with equal or greater potency than for RGS4. The polypharmacology of known RGS inhibitors may reflect their cysteine-dependent inhibition mechanism, and for this reason, none of these identified inhibitors affect the $\mathrm{R} 7$ members RGS6 and RGS7, which lack reactive Cys in their RGS domains. In addition, targeting of intracellular RGS protein-protein interactions has proven extremely challenging (reviewed by Neubig and Siderovski [2002]).

In recent years, limitations in both pharmacological compound selectivity and therapeutic benefits in PD have prompted the search for and development of novel nonpharmacological therapies. One of the most recent nonpharmacological approaches to emerge in PD treatment is gene therapy. Gene therapy entails viral delivery of genetic material to a patient either to modify (i.e., activate or suppress) endogenous gene expression or to introduce exogenous genes. Both AAV and lentiviral approaches, which are attractive due to their longterm expression efficacy and lack of immunogenicity, have been investigated in clinical trials for PD treatment in humans (reviewed by Hitti et al. [2019]). Currently, clinical trials utilizing gene therapy for PD treatment are aimed at enhancing DA synthesis (AAV-aromatic L-amino acid decarboxylase), promoting neuronal survival via enhanced neurotrophic factor expression (AAV-neurturin), or promoting proper motor function through modification of basal ganglia signaling (AAV-glutamate decarboxylase) (Muramatsu, 2010; Elkouzi et al., 2019; Hitti et al., 2019). Finally, the use of CRISPR is also now under investigation to modulate gene function in the mammalian brain (Swiech et al., 2015; Heidenreich and Zhang, 2016; Zhou et al., 2018) that may prove useful in PD therapeutics.

As discussed above, although RGSs 4, 6, 9, and 10 have been shown to be critical modulators or SNc DA neuron survival and/or motor function, the difficulty in creating selective activating (RGSs 6, 9, and 10) and inhibitory (RGS4) compounds limits their pharmacological usefulness. Therefore, future studies should focus on developing novel gene therapy approaches to selectively enhance (RGS6, RGS9, and RGS10) or diminish (RGS4) RGS protein signaling in the SNc (RGS6 and RGS10) or striatum (RGS9 and RGS4) of patients with PD. Such strategies may provide new PD therapies that not only work to correct symptomology but that also prevent pathology.

\section{Authorship Contributions}

Wrote or contributed to the writing of the manuscript: AhlersDannen, Spicer, Fisher.

\section{References}

Ashrafi A, Garcia P, Kollmus H, Schughart K, Del Sol A, Buttini M, and Glaab E (2017) Absence of regulator of G-protein signaling 4 does not protect against dopamine neuron dysfunction and injury in the mouse 6-hydroxydopamine lesion model of Parkinson's disease. Neurobiol Aging 58:30-33.

Bansal G, Druey KM, and Xie Z (2007) R4 RGS proteins: regulation of G-protein signaling and beyond. Pharmacol Ther 116:473-495.

Bello EP, Mateo Y, Gelman DM, Noaín D, Shin JH, Low MJ, Alvarez VA, Lovinger $\mathrm{DM}$, and Rubinstein M (2011) Cocaine supersensitivity and enhanced motivation for reward in mice lacking dopamine D2 autoreceptors. Nat Neurosci 14: 1033-1038.

Bifsha P, Yang J, Fisher RA, and Drouin J (2014) Rgs6 is required for adult maintenance of dopaminergic neurons in the ventral substantia nigra. PLoS Genet 10: e1004863.

Blazer LL, Storaska AJ, Jutkiewicz EM, Turner EM, Calcagno M, Wade SM, Wang Q, Huang XP, Traynor JR, Husbands SM, et al. (2015) Selectivity and antiParkinson's potential of thiadiazolidinone RGS4 inhibitors. ACS Chem Neurosci 6 : 911-919.

Chartier-Harlin MC, Kachergus J, Roumier C, Mouroux V, Douay X, Lincoln S, Levecque C, Larvor L, Andrieux J, Hulihan M, et al. (2004) Alpha-synuclein locus duplication as a cause of familial Parkinson's disease. Lancet 364:1167-1169.

Chatterjee TK, Liu Z, and Fisher RA (2003) Human RGS6 gene structure, complex alternative splicing, and role of $\mathrm{N}$ terminus and $\mathrm{G}$ protein gamma-subunit-like (GGL) domain in subcellular localization of RGS6 splice variants. J Biol Chem 278: 30261-30271.

Chaudhuri KR, Healy DG, and Schapira AH; National Institute for Clinical Excellence (2006) Non-motor symptoms of Parkinson's disease: diagnosis and management. Lancet Neurol 5:235-245.

Cheever ML, Snyder JT, Gershburg S, Siderovski DP, Harden TK, and Sondek J (2008) Crystal structure of the multifunctional Gbeta5-RGS9 complex. Nat Struct Mol Biol 15:155-162.

Chen CK, Eversole-Cire P, Zhang H, Mancino V, Chen YJ, He W, Wensel TG, and Simon MI (2003) Instability of GGL domain-containing RGS proteins in mice lacking the G protein beta-subunit Gbeta5. Proc Natl Acad Sci USA 100: 6604-6609.

Chu Y and Kordower JH (2007) Age-associated increases of alpha-synuclein in monkeys and humans are associated with nigrostriatal dopamine depletion: is this the target for Parkinson's disease? Neurobiol Dis 25:134-149.

Ding J, Guzman JN, Tkatch T, Chen S, Goldberg JA, Ebert PJ, Levitt P, Wilson CJ, Hamm HE, and Surmeier DJ (2006) RGS4-dependent attenuation of M4 autoreceptor function in striatal cholinergic interneurons following dopamine depletion. Nat Neurosci 9:832-842.

Drenan RM, Doupnik CA, Jayaraman M, Buchwalter AL, Kaltenbronn KM, Huettner JE, Linder ME, and Blumer KJ (2006) R7BP augments the function of RGS7*Gbeta5 complexes by a plasma membrane-targeting mechanism. J Biol Chem 281:28222-28231.

Ebert PJ, Campbell DB, and Levitt P (2006) Bacterial artificial chromosome transgenic analysis of dynamic expression patterns of regulator of G-protein signaling 4 during development. I. Cerebral cortex. Neuroscience 142:1145-1161.

Elkouzi A, Vedam-Mai V, Eisinger RS, and Okun MS (2019) Emerging therapies in Parkinson disease - repurposed drugs and new approaches. Nat Rev Neurol 15: 204-223.

Erdely HA, Lahti RA, Lopez MB, Myers CS, Roberts RC, Tamminga CA, and Vogel MW (2004) Regional expression of RGS4 mRNA in human brain. Eur $J$ Neurosci 19:3125-3128.

Fahn S (2008) The history of dopamine and levodopa in the treatment of Parkinson's disease. Mov Disord 23 (Suppl 3):S497-S508.

Fearnley JM and Lees AJ (1991) Ageing and Parkinson's disease: substantia nigra regional selectivity. Brain 114:2283-2301.

Geurts M, Maloteaux JM, and Hermans E (2003) Altered expression of regulators of G-protein signaling (RGS) mRNAs in the striatum of rats undergoing dopamine depletion. Biochem Pharmacol 66:1163-1170.

Giasson BI, Duda JE, Quinn SM, Zhang B, Trojanowski JQ, and Lee VM (2002) Neuronal alpha-synucleinopathy with severe movement disorder in mice expressing A53T human alpha-synuclein. Neuron 34:521-533.

Gold SJ, Hoang CV, Potts BW, Porras G, Pioli E, Kim KW, Nadjar A, Qin C, LaHoste GJ, Li Q, et al. (2007) RGS9-2 negatively modulates L-3,4-dihydroxyphenylalanineinduced dyskinesia in experimental Parkinson's disease. $J$ Neurosci 27: 14338-14348.

Gold SJ, Ni YG, Dohlman HG, and Nestler EJ (1997) Regulators of G-protein signaling (RGS) proteins: region-specific expression of nine subtypes in rat brain. J Neurosci 17:8024-8037.

Hayes MP, Bodle CR, and Roman DL (2018) Evaluation of the selectivity and cysteine dependence of inhibitors across the regulator of $\mathrm{G}$ protein-signaling family. Mol Pharmacol 93:25-35.

He W, Cowan CW, and Wensel TG (1998) RGS9, a GTPase accelerator for phototransduction. Neuron 20:95-102. 
Heidenreich M and Zhang F (2016) Applications of CRISPR-Cas systems in neuroscience. Nat Rev Neurosci 17:36-44.

Hitti FL, Yang AI, Gonzalez-Alegre P, and Baltuch GH (2019) Human gene therapy approaches for the treatment of Parkinson's disease: an overview of current and completed clinical trials. Parkinsonism Relat Disord 66:16-24

Hooks SB, Waldo GL, Corbitt J, Bodor ET, Krumins AM, and Harden TK (2003) RGS6, RGS7, RGS9, and RGS11 stimulate GTPase activity of Gi family G-proteins with differential selectivity and maximal activity. J Biol Chem 278:10087-10093.

Hunt TW, Fields TA, Casey PJ, and Peralta EG (1996) RGS10 is a selective activator of G alpha i GTPase activity. Nature 383:175-177.

Jakobsdottir J, Conley YP, Weeks DE, Mah TS, Ferrell RE, and Gorin MB (2005) Susceptibility genes for age-related maculopathy on chromosome 10q26. Am $J$ Hum Genet 77:389-407.

Jenner P (2013) Wearing off, dyskinesia, and the use of continuous drug delivery in Parkinson's disease. Neurol Clin 31 (3 Suppl):S17-S35.

Joers V, Tansey MG, Mulas G, and Carta AR (2017) Microglial phenotypes in Parkinson's disease and animal models of the disease. Prog Neurobiol 155:57-75.

Kannarkat GT, Lee JK, Ramsey CP, Chung J, Chang J, Porter I, Oliver D, Shepherd $\mathrm{K}$, and Tansey MG (2015) Age-related changes in regulator of G-protein signaling (RGS)-10 expression in peripheral and central immune cells may influence the risk for age-related degeneration. Neurobiol Aging 36:1982-1993.

Kim HJ (2013) Alpha-synuclein expression in patients with Parkinson's disease: a clinician's perspective. Exp Neurobiol 22:77-83.

Ko WK, Martin-Negrier ML, Bezard E, Crossman AR, and Ravenscroft P (2014) RGS4 is involved in the generation of abnormal involuntary movements in the unilateral 6-OHDA-lesioned rat model of Parkinson's disease. Neurobiol Dis 70 138-148.

Kovoor A, Seyffarth P, Ebert J, Barghshoon S, Chen CK, Schwarz S, Axelrod JD, Cheyette BN, Simon MI, Lester HA, et al. (2005) D2 dopamine receptors colocalize regulator of G-protein signaling 9-2 (RGS9-2) via the RGS9 DEP domain, and RGS9 knock-out mice develop dyskinesias associated with dopamine pathways. J Neurosci 25:2157-2165.

Lee JK, Chung J, Druey KM, and Tansey MG (2012) RGS10 exerts a neuroprotective role through the PKA/c-AMP response-element (CREB) pathway in dopaminergic neuron-like cells. J Neurochem 122:333-343.

Lee JK, Chung J, McAlpine FE, and Tansey MG (2011) Regulator of G-protein signaling-10 negatively regulates NF-кB in microglia and neuroprotects dopaminergic neurons in hemiparkinsonian rats. $J$ Neurosci 31:11879-11888.

Lee JK, McCoy MK, Harms AS, Ruhn KA, Gold SJ, and Tansey MG (2008) Regulator of G-protein signaling 10 promotes dopaminergic neuron survival via regulation of the microglial inflammatory response. J Neurosci 28:8517-8528.

Lemos A, Melo R, Preto AJ, Almeida JG, Moreira IS, and Dias Soeiro Cordeiro MN (2018) In silico studies targeting G-protein coupled receptors for drug research against Parkinson's disease. Curr Neuropharmacol 16:786-848.

Lerner TN and Kreitzer AC (2012) RGS4 is required for dopaminergic control of striatal LTD and susceptibility to parkinsonian motor deficits. Neuron 73:347-359.

Li W, Lesuisse C, Xu Y, Troncoso JC, Price DL, and Lee MK (2004) Stabilization of alpha-synuclein protein with aging and familial Parkinson's disease-linked A53T mutation. J Neurosci 24:7400-7409.

Lindgren N, Usiello A, Goiny M, Haycock J, Erbs E, Greengard P, Hokfelt T, Borrelli $\mathrm{E}$, and Fisone G (2003) Distinct roles of dopamine D2L and D2S receptor isoform in the regulation of protein phosphorylation at presynaptic and postsynaptic sites. Proc Natl Acad Sci USA 100:4305-4309.

Liou YJ, Chen ML, Wang YC, Chen JY, Liao DL, Bai YM, Lin CC, Chen TT, Mo GH, and Lai IC (2009) Analysis of genetic variations in the RGS9 gene and antipsychotic-induced tardive dyskinesia in schizophrenia. Am J Med Genet B Neuropsychiatr Genet 150B:239-242.

Luo Z, Ahlers-Dannen KE, Spicer MM, Yang J, Alberico S, Stevens HE, Narayanan NS, and Fisher RA (2019) Age-dependent nigral dopaminergic neurodegeneration and $\alpha$-synuclein accumulation in RGS6-deficient mice. JCI Insight 5 (13), doi: 10.1172/jci.insight.126769.

Maity B, Stewart A, Yang J, Loo L, Sheff D, Shepherd AJ, Mohapatra DP, and Fisher RA (2012) Regulator of G protein signaling 6 (RGS6) protein ensures coordination of motor movement by modulating GABAB receptor signaling. J Biol Chem 287 $4972-4981$

Maity B, Yang J, Huang J, Askeland RW, Bera S, and Fisher RA (2011) Regulator of $\mathrm{G}$ protein signaling 6 (RGS6) induces apoptosis via a mitochondrial-dependent pathway not involving its GTPase-activating protein activity. J Biol Chem 286 1409-1419

Martemyanov KA, Yoo PJ, Skiba NP, and Arshavsky VY (2005) R7BP, a novel neuronal protein interacting with RGS proteins of the R7 family. $J$ Biol Chem 280: 5133-5136.

Masliah E, Rockenstein E, Veinbergs I, Mallory M, Hashimoto M, Takeda A, Sagara Y, Sisk A, and Mucke L (2000) Dopaminergic loss and inclusion body formation in alpha-synuclein mice: implications for neurodegenerative disorders. Science $\mathbf{2 8 7}$ $1265-1269$

McCoy MK, Martinez TN, Ruhn KA, Szymkowski DE, Smith CG, Botterman BR, Tansey KE, and Tansey MG (2006) Blocking soluble tumor necrosis factor signaling with dominant-negative tumor necrosis factor inhibitor attenuates loss of dopaminergic neurons in models of Parkinson's disease. J Neurosci 26 9365-9375

McCoy MK, Ruhn KA, Martinez TN, McAlpine FE, Blesch A, and Tansey MG (2008) Intranigral lentiviral delivery of dominant-negative TNF attenuates neurodegeneration and behavioral deficits in hemiparkinsonian rats. Mol Ther 16 1572-1579.

Meissner WG, Frasier M, Gasser T, Goetz CG, Lozano A, Piccini P, Obeso JA, Rascol O, Schapira A, Voon V, et al. (2011) Priorities in Parkinson's disease research. Nat Rev Drug Discov 10:377-393.

Mhyre TR, Boyd JT, Hamill RW, and Maguire-Zeiss KA (2012) Parkinson's disease Subcell Biochem 65:389-455.
Mittal S, Bjørnevik K, Im DS, Flierl A, Dong X, Locascio JJ, Abo KM, Long E, Jin M, $\mathrm{Xu} \mathrm{B}$, et al. (2017) $\beta 2$-Adrenoreceptor is a regulator of the $\alpha$-synuclein gene driving risk of Parkinson's disease. Science 357:891-898.

Muramatsu S (2010) The current status of gene therapy for Parkinson's disease. Ann Neurosci 17:92-95.

Narayanan V, Sandiford SL, Wang Q, Keren-Raifman T, Levay K, and Slepak VZ (2007) Intramolecular interaction between the DEP domain of RGS7 and the Gbeta5 subunit. Biochemistry 46:6859-6870.

Neubig RR and Siderovski DP (2002) Regulators of G-protein signalling as new central nervous system drug targets. Nat Rev Drug Discov 1:187-197.

Nussbaum RL and Ellis CE (2003) Alzheimer's disease and Parkinson's disease. $N$ Engl $J$ Med 348:1356-1364.

O’Brien JB, Wilkinson JC, and Roman DL (2019) Regulator of G-protein signaling (RGS) proteins as drug targets: progress and future potentials. J Biol Chem 294: 18571-18585.

Pahwa R and Lyons KE (2009) Levodopa-related wearing-off in Parkinson's disease: identification and management. Curr Med Res Opin 25:841-849.

Poewe W (2008) Non-motor symptoms in Parkinson's disease. Eur J Neurol 15 (Suppl 1):14-20.

Porter MY, Xie K, Pozharski E, Koelle MR, and Martemyanov KA (2010) A conserved protein interaction interface on the type $5 \mathrm{G}$ protein beta subunit controls proteolytic stability and activity of $\mathrm{R} 7$ family regulator of $\mathrm{G}$ protein signaling proteins. $J$ Biol Chem 285:41100-41112.

Posner BA, Gilman AG, and Harris BA (1999) Regulators of G protein signaling 6 and 7. Purification of complexes with gbeta5 and assessment of their effects on $\mathrm{g}$ protein-mediated signaling pathways. J Biol Chem 274:31087-31093.

Rahman Z, Gold SJ, Potenza MN, Cowan CW, Ni YG, He W, Wensel TG, and Nestler EJ (1999) Cloning and characterization of RGS9-2: a striatal-enriched alternatively spliced product of the RGS9 gene. J Neurosci 19:2016-2026.

Rahman Z, Schwarz J, Gold SJ, Zachariou V, Wein MN, Choi KH, Kovoor A, Chen CK, DiLeone RJ, Schwarz SC, et al. (2003) RGS9 modulates dopamine signaling in the basal ganglia. Neuron 38:941-952.

Schmidt S, Hauser MA, Scott WK, Postel EA, Agarwal A, Gallins P, Wong F, Chen YS, Spencer K, Schnetz-Boutaud N, et al. (2006) Cigarette smoking strongly modifies the association of LOC387715 and age-related macular degeneration. Am $J$ Hum Genet 78:852-864.

Schrag A and Quinn N (2000) Dyskinesias and motor fluctuations in Parkinson's disease. A community-based study. Brain 123:2297-2305

Shulman JM, De Jager PL, and Feany MB (2011) Parkinson's disease: genetics and pathogenesis. Annu Rev Pathol 6:193-222.

Singleton AB, Farrer M, Johnson J, Singleton A, Hague S, Kachergus J, Hulihan M, Peuralinna T, Dutra A, Nussbaum R, et al. (2003) alpha-Synuclein locus triplication causes Parkinson's disease. Science 302:841.

Snow BE, Betts L, Mangion J, Sondek J, and Siderovski DP (1999) Fidelity of G protein beta-subunit association by the G protein gamma-subunit-like domains of RGS6, RGS7, and RGS11. Proc Natl Acad Sci USA 96:6489-6494.

Spillantini MG, Crowther RA, Jakes R, Hasegawa M, and Goedert M (1998) alphaSynuclein in filamentous inclusions of Lewy bodies from Parkinson's disease and dementia with lewy bodies. Proc Natl Acad Sci USA 95:6469-6473.

Spillantini MG, Schmidt ML, Lee VM, Trojanowski JQ, Jakes R, and Goedert M (1997) Alpha-synuclein in Lewy bodies. Nature 388:839-840.

Stefanis L (2012) $\alpha$-Synuclein in Parkinson's disease. Cold Spring Harb Perspect Med 2:a009399.

Stewart A, Maity B, Anderegg SP, Allamargot C, Yang J, and Fisher RA (2015) Regulator of $\mathrm{G}$ protein signaling 6 is a critical mediator of both reward-related behavioral and pathological responses to alcohol. Proc Natl Acad Sci USA 112 : E786-E795.

Stewart A, Maity B, Wunsch AM, Meng F, Wu Q, Wemmie JA, and Fisher RA (2014) Regulator of G-protein signaling 6 (RGS6) promotes anxiety and depression by attenuating serotonin-mediated activation of the 5-HT(1A) receptor-adenylyl cyclase axis. FASEB $J$ 28:1735-1744.

Subhramanyam CS, Wang C, Hu Q, and Dheen ST (2019) Microglia-mediated neuroinflammation in neurodegenerative diseases. Semin Cell Dev Biol 94:112-120.

Swiech L, Heidenreich M, Banerjee A, Habib N, Li Y, Trombetta J, Sur M, and Zhang $\mathrm{F}$ (2015) In vivo interrogation of gene function in the mammalian brain using CRISPR-Cas9. Nat Biotechnol 33:102-106.

Tansey MG and Goldberg MS (2010) Neuroinflammation in Parkinson's disease: its role in neuronal death and implications for therapeutic intervention. Neurobiol Dis 37 (3):510-518.

Tekumalla PK, Calon F, Rahman Z, Birdi S, Rajput AH, Hornykiewicz O, Di Paolo T, Bédard PJ, and Nestler EJ (2001) Elevated levels of DeltaFosB and RGS9 in striatum in Parkinson's disease. Biol Psychiatry 50:813-816.

Thanvi B, Lo N, and Robinson T (2007) Levodopa-induced dyskinesia in Parkinson's disease: clinical features, pathogenesis, prevention and treatment. Postgrad Med $J$ 83:384-388.

Thomas EA, Danielson PE, and Sutcliffe JG (1998) RGS9: a regulator of G-protein signalling with specific expression in rat and mouse striatum. J Neurosci Res 52:118-124 Usiello A, Baik JH, Rougé-Pont F, Picetti R, Dierich A, LeMeur M, Piazza PV, and Borrelli E (2000) Distinct functions of the two isoforms of dopamine D2 receptors. Nature 408:199-203.

Wang Y, Xu R, Sasaoka T, Tonegawa S, Kung MP, and Sankoorikal EB (2000) Dopamine D2 long receptor-deficient mice display alterations in striatum-dependent functions, $J$ Neurosci $20 \cdot 8305-8314$.

Waugh JL, Lou AC, Eisch AJ, Monteggia LM, Muly EC, and Gold SJ (2005) Regional, cellular, and subcellular localization of RGS10 in rodent brain. J Comp Neurol 481: 299-313.

Witherow DS, Wang Q, Levay K, Cabrera JL, Chen J, Willars GB, and Slepak VZ (2000) Complexes of the $\mathrm{G}$ protein subunit gbeta 5 with the regulators of $\mathrm{G}$ protein signaling RGS7 and RGS9. Characterization in native tissues and in transfected cells. J Biol Chem 275:24872-24880. 
Yang J, Huang J, Maity B, Gao Z, Lorca RA, Gudmundsson H, Li J, Stewart A, Swaminathan PD, Ibeawuchi SR, et al. (2010) RGS6, a modulator of parasympathetic activation in heart. Circ Res 107:1345-1349.

Zhang K, Howes KA, He W, Bronson JD, Pettenati MJ, Chen C, Palczewski K, Wensel TG, and Baehr W (1999) Structure, alternative splicing, and expression of the human RGS9 gene. Gene 240:23-34.

Zhang S, Watson N, Zahner J, Rottman JN, Blumer KJ, and Muslin AJ (1998) RGS3 and RGS4 are GTPase activating proteins in the heart. $J$ Mol Cell Cardiol 30: $269-276$
Zhou H, Liu J, Zhou C, Gao N, Rao Z, Li H, Hu X, Li C, Yao X, Shen X, et al. (2018) In vivo simultaneous transcriptional activation of multiple genes in the brain using CRISPR-dCas9-activator transgenic mice. Nat Neurosci 21:440-446.

Address correspondence to: Rory A. Fisher, Department of Neuroscience and Pharmacology, University of Iowa, Carver College of Medicine, 51 Newton Rd., BSB 2-512, Iowa City, IA 52242. E-mail: rory-fisher@uiowa.edu 